Proyecciones Journal of Mathematics Vol. 31, No 2, pp. 149-164, June 2012. Universidad Católica del Norte Antofagasta - Chile

\title{
Uniform Convergence and the Hahn-Schur Theorem
}

\author{
CHARLES SWARTZ \\ NEW MEXICO STATE UNIVERSITY, U.S.A. \\ Received : January 2012. Accepted : February 2012
}

\begin{abstract}
Let $E$ be a vector space, $F$ a set, $G$ be a locally convex space, $b: E \times F \rightarrow G$ a map such that $b(\cdot, y): E \rightarrow G$ is linear for every $y \in F$; we write $b(x, y)=x \cdot y$ for brevity. Let $\lambda$ be a scalar sequence space and $w(E, F)$ the weakest topology on $E$ such that the linear maps $b(\cdot, y): E \rightarrow G$ are continuous for all $y \in F$. A series $\sum_{j} x_{j}$ in $X$ is $\lambda$ multiplier convergent with respect to $w(E, F)$ if for each $t=\left\{t_{j}\right\} \in \lambda$, the series $\sum_{j=1}^{\infty} t_{j} x_{j}$ is $w(E, F)$ convergent in $E$. For multiplier spaces $\lambda$ satisfying certain gliding hump properties we establish the following uniform convergence result: Suppose $\sum_{j} x_{i j}$ is $\lambda$ multiplier convergent with respect to $w(E, F)$ for each $i \in \mathbf{N}$ and for each $t \in \lambda$ the set $\left\{\sum_{j=1}^{\infty} t_{j} x_{i j}: i\right\}$ is uniformly bounded on any subset $B \subset F$ such that $\{x \cdot y: y \in B\}$ is bounded for $x \in E$. Then for each $t \in \lambda$ the series $\sum_{j=1}^{\infty} t_{j} x_{i j} \cdot y$ converge uniformly for $y \in B, i \in \mathbf{N}$. This result is used to prove a Hahn-Schur Theorem for series such that $\lim _{i} \sum_{j=1}^{\infty} t_{j} x_{i j} \cdot y$ exists for $t \in \lambda, y \in F$. Applications of these abstract results are given to spaces of linear operators, vector spaces in duality, spaces of continuous functions and spaces with Schauder bases.
\end{abstract}

Key Words : Multiplier convergent series, uniform convergence, Hahn-Schur Theorem. 
One version of the scalar Hahn-Schur Theorem ([Ha], [Sc]) asserts that a sequence in $l^{1}$ which is weakly convergent is norm convergent even though the weak topology is strictly weaker than the norm topology ([K1] 22.4.(2), [Sw1] 16.14, [Wi] 14.4.7 ]). The result has been strengthened and generalized in many directions (see Chapters 8 and 9 of [Sw2]). In particular, there have been versions for subseries convergent series and bounded multiplier convergent series in topological vector spaces([Sw2] Chapter 8). In [CL] two different gliding hump properties have been used to derive abstract versions of the Orlicz-Pettis Theorem for multiplier convergent series with respect to weak and strong topologies. In this paper we show that these two gliding hump properties can also be employed to derive versions of the Hahn-Schur Theorem for multiplier convergent series with respect to weak and strong topologies. We first establish an abstract result for uniform convergence of multiplier convergent series with respect to a bilinear type operator and then use this result to establish abstract versions of the Hahn-Schur Theorem. These results are referred to as Hahn-Schur theorems because weakly convergent sequences are shown to converge in stronger topologies. This result is then applied to obtain versions of the Hahn-Schur Theorem for operator valued and vector valued series.

We begin by describing the abstract setting which will be used to establish the initial results. Similar settings have been employed in [BCS], [CL], and [Sw4]. Let $E$ be a vector space, $F$ a set, $G$ a Hausdorff locally convex space and $b: E \times F \rightarrow G$ a map such that $b(\cdot, y): E \rightarrow G$ is linear for $y \in F$; for brevity we often write $b(x, y)=x \cdot y$ for $x \in E, y \in F$. Let $w(E, F)$ be the weakest topology on $E$ such that the linear maps $b(\cdot, y): E \rightarrow G$ are continuous for every $y \in F$. In many applications of this setting the set $F$ is a vector space and $b$ is a bilinear map; this is the case in the references above. We give two examples where $b$ is bilinear and which are used in the applications of the abstract results; examples where $F$ is just a set are described in the last two results.

Example 1. Let $X, Y$ be Hausdorff locally convex spaces and $L(X, Y)$ the space of all continuous linear operators from $X$ into $Y$. The bilinear map we consider is the map $b: L(X, Y) \times X \rightarrow Y$ defined by $b(T, x)=T x$. In this case the topology $w(L(X, Y), X)$ is just the strong operator topology which we denote by $L_{s}(X, Y)$. 
Example 2. Let $E, E^{\prime}$ be a pair of vector spaces in duality with respect to the bilinear pairing $\langle$,$\rangle . We consider b\left(x, x^{\prime}\right)=\left\langle x^{\prime}, x\right\rangle$; then $w\left(E, E^{\prime}\right)=$ $\sigma\left(E, E^{\prime}\right)$, the weak topology.

Let $\lambda$ be a sequence space containing the space $c_{00}$ of all sequences which are eventually 0 . If $Z$ is a topological vector space, a series $\sum_{j} z_{j}$ in $Z$ is $\lambda$ multiplier convergent in $Z$ if the series $\sum_{j=1}^{\infty} t_{j} z_{j}$ converges in $Z$ for every $t=\left\{t_{j}\right\} \in \lambda$. If $\lambda=m_{0}$, the space of all sequences with finite range, then $m_{0}$ multiplier convergent series are just the subseries convergent series; if $\lambda=l^{\infty}$, the $l^{\infty}$ multiplier convergent series are often called the bounded multiplier convergent series.

We describe the gliding hump properties which will be employed. If $t=\left\{t_{j}\right\}, s=\left\{s_{j}\right\}$ are scalar sequences, st will denote the coordinatewise product of $s$ and $t$; if $I \subset \mathbf{N}, \chi_{I}$ will denote the characteristic function of $I$.

Definition 3. $\lambda$ is monotone if $\chi_{I} t \in \lambda$ for every $I \subset \mathbf{N}$ and $t \in \lambda$.

For example, $l^{p}, 0<p \leq \infty, m_{0}, c_{0}$ are monotone. Further examples can be found in Appendix B of [Sw3].

Definition 4. $\lambda$ is $c_{0}$-factorable if each $t \in \lambda$ can be written as $t=s u$ with $s \in c_{0}$ and $u \in \lambda$ (this property has been also referred to as $c_{0}$-invariant ([Ga]) and $c_{0}$-decomposable $\left.([L W])\right)$.

For example, $l^{p}, 0<p<\infty, c_{0}, c s$ are $c_{0}$-factorable. Further examples can be found in Appendix B of [Sw3].

Definition 5. The space $\lambda$ has the infinite gliding hump property $(\infty-$ $G H P$ ) if whenever $t \in \lambda$ and $\left\{I_{j}\right\}$ is an increasing sequence of intervals there exist a subsequence $\left\{n_{j}\right\}$ and $a_{n_{j}}>0, a_{n_{j}} \rightarrow \infty$ such that every subsequence of $\left\{n_{j}\right\}$ has a further subsequence $\left\{p_{j}\right\}$ such that the coordinate $\operatorname{sum} \sum_{j=1}^{\infty} a_{p_{j}} \chi_{I_{p_{j}}} t \in \lambda$.

For example, $l^{p}, 0<p<\infty$, and $c s$ have $\infty-G H P$. Further examples can be found in Appendix B of [Sw3].

For the convenience of the reader we state two of the results which will be used in the proof below. First, an interesting lemma of Li/Wang ([LW]Lemma 3.2). 
Lemma 6. Let $Z$ be a vector space and $K \subset Z$ a convex subset which contains 0 . If $x_{1}, \ldots, x_{n} \in K$ and $M>0$ is such that $M \sum_{j \in \Delta} x_{j} \in K$ for every $\Delta \subset\{1, \ldots, n\}$, then $\sum_{j=1}^{n} s_{j} x_{j} \in K$ for every $0 \leq s_{j} \leq M, j=1, \ldots, n$.

The other result is the Antosik-Mikusinski Matrix Theorem. We state a version which will be used; more general forms can be found in [Sw1] 9.2, [Sw2] Appendix D.

Theorem 7. (Antosik-Mikusinski) Let $x_{i j} \in G$ for $i, j \in \mathbf{N}$. Assume (1) $\lim _{i} x_{i j}=x_{j}$ exists for every $j$ and (2) for every increasing sequence of positive integers $\left\{m_{j}\right\}$ there is a subsequence $\left\{n_{j}\right\}$ of $\left\{m_{j}\right\}$ such that the series $\sum_{j=1}^{\infty} x_{i n_{j}}$ converges and $\lim _{i} \sum_{j=1}^{\infty} x_{i n_{j}}$ exists. Then $\lim _{i} x_{i i}=0$.

Definition 8. A subset $B \subset F$ is pointwise bounded if $\{b(x, y): y \in B\}$ is bounded for every $x \in E$.

In what follows, if the series $\sum_{j} x_{j}$ is $\lambda$ multiplier convergent with respect to $w(E, F), \sum_{j=1}^{\infty} t_{j} x_{j}$ will denote the $w(E, F)$ sum of the series when $t=\left\{t_{j}\right\} \in \lambda$.

Theorem 9. Suppose $\lambda$ is either $c_{0}$-factorable and monotone or has $\infty$ $G H P$ and that $\sum_{j} x_{i j}$ is $\lambda$ multiplier convergent with respect to $w(E, F)$ for every $i \in \mathbf{N}$. If

(\#) for every $t \in \lambda\left\{\sum_{j=1}^{\infty} t_{j} x_{i j}: i \in \mathbf{N}\right\}$ is uniformly bounded on the pointwise bounded set $B \subset F$

(that is, $\left\{\sum_{j=1}^{\infty} t_{j} x_{i j} \cdot y: i \in \mathbf{N}, y \in B\right\}$ is bounded in $G$ ), then (\#\#) for every $t \in \lambda$ the series $\sum_{j=1}^{\infty} t_{j} x_{i j} \cdot y$ converge uniformly for

$\in \mathbf{N}, y \in B$.

Proof : If the conclusion fails to hold, there exists a convex neighborhood of 0 in $G, W$, such that for every $n$ there exist $k_{n}, y_{n} \in B$, an interval $I_{n}$ with $\min I_{n}>n$ such that $\sum_{l \in I_{n}} t_{l} x_{k_{n} l} \cdot y_{n} \notin W$. Applying this condition to $n=1$, there exist $k_{1}, y_{1} \in B$, an interval $I_{1}$ with $\min I_{1}>1$ such that $\sum_{l \in I_{1}} t_{l} x_{k_{1} l} \cdot y_{1} \notin W$. By the Orlicz-Pettis Theorem given in [CL] (Theorem 
1 for the case when $\lambda$ is $c_{0}$-factorable and monotone and Theorem 5 for the case when $\lambda$ has $\infty$-GHP; the proofs of these results do not require that $F$ be a vector space and $b$ be bilinear), each series $\sum_{j=1}^{\infty} t_{j} x_{i j} \cdot y$ converges uniformly for $y \in B$ so there exists $m^{\prime}>\max I_{1}$ such that $\sum_{j=p}^{q} t_{j} x_{i j} \cdot y \in W$ for $q>p \geq m^{\prime}, 1 \leq i \leq k_{1}, y \in B$. Applying the condition above to $m^{\prime}$ there exist $k_{2}, y_{2} \in B$, an interval $I_{2}$ with $\min I_{2}>m^{\prime}$ such that $\sum_{l \in I_{2}} t_{l} x_{k_{2} l} \cdot y_{2} \notin W$. Note $k_{2}>k_{1}$. Continuing this construction produces an increasing sequence $\left\{k_{i}\right\},\left\{y_{i}\right\} \subset B$, intervals $\left\{I_{i}\right\}$ with $\max I_{i}<\min I_{i+1}$ such that

$$
\text { (\$) } \sum_{l \in I_{i}} t_{l} x_{k_{i} l} \cdot y_{i} \notin W \text { for every } i \text {. }
$$

Consider first the case when $\lambda$ is $c_{0}$-factorable and monotone. Since $\lambda$ is $c_{0}$-factorable, $t=s u$ with $s \in c_{0}$ and $u \in \lambda$ and since $\lambda$ is monotone we may assume that $s \geq 0$. Then $\sum_{l \in I_{i}} s_{l} u_{l} x_{k_{i} l} \cdot y_{i} \notin W$. Set $r_{i}=\max \left\{s_{l}: l \in I_{i}\right\}$ so $r_{i} \rightarrow 0$. Lemma 6 implies there exists $\Delta_{i} \subset I_{i}$ with

$$
\text { (*) } r_{i} \sum_{l \in \Delta_{i}} u_{l} x_{k_{i} l} \cdot y_{i} \notin W .
$$

Define a matrix

$$
M=\left[m_{i j}\right]=\left[r_{i} \sum_{l \in \Delta_{j}} u_{l} x_{k_{i} l} \cdot y_{i}\right] .
$$

We show that $M$ satisfies the conditions of the Antosik-Mikusinski Theorem. First, (\#) with $t=\chi_{\Delta_{j}} u$ implies $\left\{\sum_{l \in \Delta_{j}} u_{i} x_{k_{i} l} \cdot y_{i}: i\right\}$ is bounded and $r_{i} \rightarrow o$ gives $\lim _{i} m_{i j}=0$. Next, $v=\left\{v_{j}\right\}=\sum_{l=1}^{\infty} \chi_{\Delta_{l}} u \in \lambda$ since $\lambda$ is monotone. Then

$$
\sum_{j=1}^{\infty} m_{i j}=\sum_{j=1}^{\infty} r_{i} \sum_{l \in \Delta_{j}} u_{i} x_{k_{i} l} \cdot y_{i}=r_{i} \sum_{l=1}^{\infty} v_{l} x_{k_{i} l} \cdot y_{i} .
$$

By (\#) $\left\{\sum_{l=1}^{\infty} v_{l} x_{k_{i} l} \cdot y_{i}: i\right\}$ is bounded and $r_{i} \rightarrow 0$ so $\lim _{i} \sum_{j=1}^{\infty} m_{i j}=0$. Since the same argument can be applied to any subsequence, the matrix $M$ satisfies the conditions of the Antosik-Mikusinski Theorem and the diagonal of $M$ converges to 0 . But, this contradicts $(\$)$. This establishes the result when $\lambda$ is $c_{0}$-factorable and monotone.

Next assume $\lambda$ has $\infty$-GHP. Then using the notation in Definition 5

$$
\sum_{j=1}^{\infty} a_{p_{j}} \chi_{I_{p_{j}}} t \in \lambda
$$


Define a matrix

$$
M=\left[m_{i j}\right]=\left[\frac{1}{a_{p_{i}}} \sum_{l \in I_{p_{j}}} a_{p_{j}} t_{l} x_{p_{i} l} \cdot y_{i}\right] .
$$

As in the proof above, we show that $M$ satisfies the conditions of the Antosik-Mikusinski Theorem. First, the columns of $M$ converge to 0 since $1 / a_{p_{i}} \rightarrow 0$ and (\#) applied to $\chi_{I_{p_{j}}} t$ implies $\left\{\sum_{l \in I_{p_{j}}} a_{p_{j}} t_{l} x_{p_{i} l} \cdot y_{i}: i\right\}$ is bounded. Given any subsequence of $\left\{p_{j}\right\}$ there exists a further subsequence $\left\{q_{j}\right\}$ such that $u=\left\{u_{j}\right\}=\sum_{j=1}^{\infty} a_{q_{j}} \chi_{I_{q_{j}}} t \in \lambda$. Then

$$
\sum_{j=1}^{\infty} m_{i q_{j}}=\frac{1}{a_{p_{i}}} \sum_{j=1}^{\infty} \sum_{l \in I_{q_{j}}} a_{q_{j}} t_{l} x_{p_{i} l} \cdot y_{i}=\frac{1}{a_{p_{i}}} \sum_{l=1}^{\infty} u_{l} x_{p_{i} l} \cdot y_{i} \rightarrow 0
$$

since $\frac{1}{a_{p_{i}}} \rightarrow 0$ and $\left\{\sum_{l=1}^{\infty} u_{l} x_{p_{i}} \cdot y_{i}: i\right\}$ is bounded by (\#). By the AntosikMikusinski Theorem the diagonal of $M$ converges to 0 contradicting (\$). This completes the proof.

Examples are given in [CL] which show that the properties " $c_{0}$-factorable and monotone" and " $\infty$-GHP" do not imply one another.

Sufficient conditions for condition (\#) to hold are given in Theorems 15 and 20 and in the remarks following Theorem 27.

Remark 10. If the multiplier space $\lambda$ also satisfies the signed strong gliding hump property (signed-SGHP), the conclusion of Theorem 9 can be strengthened. The space $\lambda$ has signed-SGHP if $\lambda$ has a vector topology under which it is a $K$-space and whenever $\left\{t^{k}\right\}$ is a bounded sequence in $\lambda$ and $\left\{I_{k}\right\}$ is an increasing sequence of intervals, there exist a sequence of signs $\left\{s_{k}\right\}$ and a subsequence $\left\{n_{k}\right\}$ such that $\sum_{k=1}^{\infty} s_{k} \chi_{I_{n_{k}}} t^{n_{k}} \in \lambda$. For example, $l^{\infty}$, and bs have signed-SGHP. Further examples can be found in Appendix $B$ of [Sw3]. The conclusion of Theorem 9 can be strengthened to read : the series $\sum_{j=1}^{\infty} t_{j} x_{i j} \cdot y$ converge uniformly for $i \in \mathbf{N}, y \in B$ and $t$ belonging to a bounded subset $A$ of $\lambda$. For if this conclusion fails to hold there exists a neighborhood $W$ of $G$ such that for every $n$ there exist $k_{n}, y_{n} \in B, t^{n} \in A$ and an interval $I_{n}$ with $\min I_{n}>n$ such that $\sum_{l \in I_{n}} t_{l}^{n} x_{k_{n} l} \cdot y_{n} \notin W$. By signed-SGHP there exist signs $\left\{s_{n}\right\}$ and a subsequence $\left\{m_{n}\right\}$ such that $t=\sum_{n=1}^{\infty} s_{n} \chi_{I_{m_{n}}} t^{m_{n}} \in \lambda$. Then $\sum_{l \in I_{m_{n}}} s_{n} t_{l} x_{k_{m_{n}}} \cdot y_{m_{n}} \notin W$ analogous to condition (\$). The proof of Theorem 9 now applies. 
Remark 11. Blasco/Calabuig/Signes ([BCS]) introduced a useful condition for treating Orlicz-Pettis Theorems with respect to bilinear mappings which is also useful in our setting. Assume that $F$ is a locally convex space and $b$ is bilinear.

$(\gamma)$ for each $x \in E, b(x, \cdot): F \rightarrow G$ is sequentially continuous.

If $(\gamma)$ is satisfied, then any bounded subset $B$ of $F$ is pointwise bounded so if $(\gamma)$ is satisfied, the conclusions of Theorems 9 hold when $B$ is a bounded subset of $F$. Note that condition $(\gamma)$ is satisfied in Example 1.

We now use Theorem 9 to establish an abstract version of the HahnSchur Theorem.

We recall a standard result.

Proposition 12. For each $i \in \mathbf{N}$ assume the series $\sum_{j=1}^{\infty} z_{i j}$ converges in $G$ and that $\lim _{i} \sum_{j=1}^{\infty} z_{i j}$ exists. If $\lim _{i} z_{i j}=z_{j}$ exists for each $j$ and the series $\sum_{j=1}^{\infty} z_{i j}$ converge uniformly for $i \in \mathbf{N}$, then the series $\sum_{j=1}^{\infty} z_{j}$ converges and $\lim _{i} \sum_{j=1}^{\infty} z_{i j}=\sum_{j=1}^{\infty} z_{j}$.

Proposition 13. Let $\sum_{j} x_{i j}$ be $\lambda$ multiplier convergent with respect to $w(E, F)$ for $i \in \mathbf{N}$ and assume $\lim _{i} \sum_{j=1}^{\infty} t_{j} x_{i j} \cdot y$ exists for $t \in \lambda, y \in F$. Let $B \subset F$. If

(1) the series $\sum_{j=1}^{\infty} t_{j} x_{i j} \cdot y$ converge uniformly for $i \in \mathbf{N}, y \in B$,

(2) for each $j$ there exists $x_{j} \in E$ such that $\lim _{i} x_{i j} \cdot y=x_{j} \cdot y$ uniformly for $y \in B$,

(3) the series $\sum_{j=1}^{\infty} t_{j} x_{j} \cdot y$ converge uniformly for $y \in B$,

then $\lim _{i} \sum_{j=1}^{\infty} t_{j} x_{i j} \cdot y=\sum_{j=1}^{\infty} t_{j} x_{j} \cdot y$ uniformly for $y \in B$.

Proof : Let $U$ be a neighborhood of 0 in $G$. Pick $V$ to be a symmetric neighborhood of 0 such that $V+V+V \subset U . \sum_{j} x_{j}$ is $\lambda$ multiplier convergent with respect to $w(E, F)$ and $\lim _{i} \sum_{j=1}^{\infty} t_{j} x_{i j} \cdot y=\sum_{j=1}^{\infty} t_{j} x_{j} \cdot y$ for each $y \in B$ by Proposition 12. By (1), (3) there exists $n$ such that $\sum_{j=n}^{\infty} t_{j} x_{i j} \cdot y \in V$ 
and $\sum_{j=n}^{\infty} t_{j} x_{j} \cdot y \in V$ for $i \in \mathbf{N}, y \in B$. Fix such an $n$. By (2) there exists $i_{0}$ such that $i \geq i_{0}$ implies $\sum_{j=1}^{n-1} t_{j}\left(x_{i j}-x_{j}\right) \cdot y \in V$ for $y \in B$. Then if $i \geq i_{0}$ and $y \in B$,

$$
\begin{gathered}
\sum_{j=1}^{\infty} t_{j} x_{i j} \cdot y-\sum_{j=1}^{\infty} t_{j} x_{j} \cdot y= \\
\sum_{j=1}^{n-1} t_{j}\left(x_{i j}-x_{j}\right) \cdot y+\sum_{j=n}^{\infty} t_{j} x_{i j} \cdot y-\sum_{j=n}^{\infty} t_{j} x_{j} \cdot y \in V+V+V \subset U .
\end{gathered}
$$

We can now give a version of the Hahn-Schur Theorem.

Theorem 14. (Hahn-Schur) Suppose $\lambda$ is either $c_{0}$-factorable and monotone or has $\infty-G H P$. Let $B \subset F$ be pointwise bounded. Let $\sum_{j} x_{i j}$ be $\lambda$ multiplier convergent with respect to $w(E, F)$ for $i \in \mathbf{N}$ and assume $\lim _{i} \sum_{j=1}^{\infty} t_{j} x_{i j} \cdot y$ exists for $t \in \lambda, y \in F$. If

(\#) for every $t \in \lambda\left\{\sum_{j=1}^{\infty} t_{j} x_{i j}: i \in \mathbf{N}\right\}$ is uniformly bounded on the pointwise bounded set $B \subset F$,

(2) for each $j$ there exists $x_{j} \in E$ such that $\lim _{i} x_{i j} \cdot y=x_{j} \cdot$ yuniformly for $\mathrm{y} \in B$, then $\sum_{j} x_{j}$ is $\lambda$ multiplier convergent with respect to $w(E, F)$ and

$$
\lim _{i} \sum_{j=1}^{\infty} t_{j} x_{i j} \cdot y=\sum_{j=1}^{\infty} t_{j} x_{j} \cdot y \text { uniformly for } y \in B .
$$

Proof : Conditions (1) and (3) of Proposition 13 hold by Theorem 9 so the result follows from Proposition 13.

Theorem 14 is referred to as a Hahn-Schur Theorem since a sequence which is assumed to converge weakly is shown to converge in a stronger sense. This is somewhat different from previous versions of the Hahn-Schur Theorem where the uniform convergence of the sequence is over subsets of the multiplier space ([Sw3]7.11) while in the theorem above the member of the multiplier space is fixed but the convergence is in a stronger topology.

We next give sufficient conditions for (\#) to hold and obtain a version of the Hahn-Schur Theorem which will be applied to operator valued series. This involves a condition similar to that of [BCS] of Remark 11. 
Assume that $F$ is a locally convex space and $b$ is a bilinear map. We say that condition $\left(\gamma^{\prime}\right)$ is satisfied if

$\left(\gamma^{\prime}\right)$ for each $x \in E$ the linear map $b(x, \cdot): F \rightarrow G$ is continuous.

Note condition $\left(\gamma^{\prime}\right)$ is satisfied in Example 1. If $\left(\gamma^{\prime}\right)$ is satisfied and $F$ is a barrelled space, then if $\left\{\sum_{j=1}^{\infty} t_{j} x_{i j}: i \in \mathbf{N}\right\}$ is pointwise bounded on $F$, the condition (\#) holds for bounded subsets of $F$ by the Uniform Boundedness principle for barrelled spaces ([K2] 39.3.(2), [Sw1] 24.11, [Wi] 9.3.4).

Using the Uniform Boundedness Principle and the Banach-Steinhaus Theorems for barrelled spaces, we can obtain a Hahn-Schur Theorem which is easier to apply to linear operators.

Theorem 15. (Hahn-Schur) Suppose $\lambda$ is either $c_{0}$-factorable and monotone or has $\infty-G H P$. Assume that condition $\left(\gamma^{\prime}\right)$ is satisfied and that $F$ is barrelled. Let $\sum_{j} x_{i j}$ be $\lambda$ multiplier convergent with respect to $w(E, F)$ for $i \in \mathbf{N}$ and assume $\lim _{i} \sum_{j=1}^{\infty} t_{j} x_{i j} \cdot y$ exists for $t \in \lambda, y \in F$. If

$\left(2^{\prime}\right)$ for each $j$ there exists $x_{j} \in E$ such that $\lim _{i} x_{i j} \cdot y=x_{j} \cdot y$ for $y \in F$,

then $\sum_{j} x_{j}$ is $\lambda$ multiplier convergent with respect to $w(E, F)$ and if $B \subset F$ is precompact,

$$
\lim _{i} \sum_{j=1}^{\infty} t_{j} x_{i j} \cdot y=\sum_{j=1}^{\infty} t_{j} x_{j} \cdot y \text { uniformly for } y \in B .
$$

Proof : The sequence of continuous linear operators $\left\{b\left(\sum_{j=1}^{\infty} t_{j} x_{i j}, \cdot\right)\right\}_{i}$ converges pointwise and is, therefore, uniformly bounded on bounded subsets by the Uniform Boundedness Principle for barrelled spaces ([K2] 39.3.(2), [Sw1] 24.11, [Wi] 9.3.4) so condition (\#) is satisfied since bounded sets are pointwise bounded. Also, from (2') and the Banach-Steinhaus Theorem ([K2] 39.5, [Sw1] 24.12) since the sequence of continuous linear operators $\left\{b\left(x_{i j}, \cdot\right)\right\}_{i}$ converge pointwise to $b\left(x_{j}, \cdot\right)$, the convergence is uniform over precompact subsets of $F$ so condition (2) is satisfied when $B$ is precompact. The result follows from Theorem 14 .

We now give applications of the abstract results to the situations covered in Examples 1 and 2. 
First consider the case of continuous linear operators as in Example 1. $L(X, Y)$ with the strong operator topology ( the topology of uniform convergence on bounded subsets of $X$, respectively, the topology of uniform convergence on precompact subsets ) will be denoted by $L_{s}(X, Y)$ $\left(L_{b}(X, Y), L p c(X, Y)\right)$. From Theorem 9, we have

Theorem 16. Suppose $\lambda$ is either $c_{0}$-factorable and monotone or has $\infty-$ GHP. Let $\sum_{j} T_{i j}$ be $\lambda$ multiplier convergent in $L_{s}(X, Y)$ for $i \in \mathbf{N}$ and

(\#) for each $t \in \lambda\left\{\sum_{j=1}^{\infty} t_{j} T_{i j}: i \in \mathbf{N}\right\}$ is uniformly bounded on the bounded subset $B \subset X$.

Then

(\#\#) the series $\sum_{j=1}^{\infty} t_{j} T_{i j} x$ converge uniformly for $i \in \mathbf{N}, x \in B$.

We now establish the version of the Hahn-Schur Theorem given in Theorem 15 for the case of continuous linear operators.

Theorem 17. Suppose $\lambda$ is either $c_{0}$-factorable and monotone or has $\infty-$ GHP and assume that $X$ is barrelled. Let $\sum_{j} T_{i j}$ be $\lambda$ multiplier convergent in $L_{s}(X, Y)$ for $i \in \mathbf{N}$. If $\lim _{i} \sum_{j=1}^{\infty} t_{j} T_{i j} x$ exists for each $t \in \lambda$ and $\lim _{i} T_{i j} x=T_{j} x$ for $x \in X$, then $\sum_{j} T_{j}$ is $\lambda$ multiplier convergent in $L_{s}(X, Y)$ and $\lim _{i} \sum_{j=1}^{\infty} t_{j} T_{i j} x=\sum_{j=1}^{\infty} t_{j} T_{j} x$ uniformly for $x$ belonging to precompact subsets of $X$, i.e., $\lim _{i} \sum_{j=1}^{\infty} t_{j} T_{i j}=\sum_{j=1}^{\infty} t_{j} T_{j}$ in $L_{p c}(X, Y)$.

Proof : Setting $t=e^{j}$, the sequence with 1 in the $j^{\text {th }}$ coordinate and 0 in the other coordinates, in the hypothesis implies that $\lim _{i} T_{i j} x=T_{j} x$ exists for each $x \in X$ and $T_{j} \in L(X, Y)$ since $X$ is barrelled ([K2] 39.5, [Sw1] 24.12, [Wi] 9.3.7). Thus, condition (2') of Theorem 15 is satisfied. Since condition $\left(\gamma^{\prime}\right)$ is satisfied, Theorem 15 is applicable and gives the result.

Remark 18. If it is the case that $\lim _{i} T_{i j}=T_{j}$ in $L_{b}(X, Y)$, then the conclusion of Theorem 17 can be strengthened to : $\lim _{i} \sum_{j=1}^{\infty} t_{j} T_{i j}=\sum_{j=1}^{\infty} t_{j} T_{j}$ in $L_{b}(X, Y)$ (Theorem 14). It should also be pointed out that the series $\sum_{j} T_{i j}, \sum_{j} T_{j}$ are $\lambda$ multiplier convergent in $L_{b}(X, Y)$ by the Orlicz-Pettis Theorems in $[C L]$ with the assumptions on the multiplier space $\lambda$. 
Remark 19. It is possible to obtain a version of the Hahn-Schur Theorem as given in Theorem 17 with weaker gliding hump assumptions on the multiplier space $\lambda$. The multiplier space $\lambda$ has the signed weak gliding hump property (signed WGHP) if whenever $t \in \lambda$ and $\left\{I_{j}\right\}$ is an increasing sequence of intervals in $\mathbf{N}$, there exist a sequence of signs $\left\{s_{j}\right\}$ and a subsequence $\left\{n_{j}\right\}$ such that the coordinate sum $\sum_{j=1}^{\infty} s_{j} \chi_{I_{n_{j}}} t \in \lambda$ (For example, any monotone space has signed-WGHP; see [Sw2], [Sw3] for further examples). Let the assumptions be as in Theorem 17 except that $\lambda$ has signed-WGHP. Then $T_{j} \in L(X, Y)$ and $\lim _{i} T_{i j} x=T_{j} x$ uniformly for $x$ belonging to precompact subsets of $X$ by the Banach-Steinhaus Theorem ([K2] 39.5, [Sw1] 24.12). We claim that $\sum_{j} T_{j}$ is $\lambda$ multiplier convergent in $L_{s}(X, Y)$. Fix $x \in X$. For each $i$ the series $\sum_{j=1}^{\infty} T_{i j} x$ is $\lambda$ multiplier convergent in $Y$ and for every $t \in \lambda, \lim _{i} \sum_{j=1}^{\infty} t_{j} T_{i j} x$ exists. By the vector version of the Hahn-Schur Theorem given in Theorem 2.28 or Theorem 7.6 of [Sw3], the series $\sum_{j}\left(\lim _{i} T_{i j} x\right)=\sum_{j} T_{j} x$ is $\lambda$ multiplier convergent and $\lim _{i} \sum_{j=1}^{\infty} t_{j} T_{i j} x=\sum_{j=1}^{\infty} t_{j} T_{j} x$. This means $\sum_{j=1}^{\infty} t_{j} T_{j} \in L(X, Y)$ where the series converges in $L_{s}(X, Y)$ by the Banach-Steinhaus Theorem. Also, by the Banach-Steinhaus Theorem, $\lim _{i} \sum_{j=1}^{\infty} t_{j} T_{i j} x=\sum_{j=1}^{\infty} t_{j} T_{j} x$ uniformly for $x$ belonging to precompact subsets of $X$. This argument does not cover the case discussed in Remark 18.

We next consider the case when $E, E^{\prime}$ are two vector spaces in duality; Example 2. The weak (strong) topology on $E$ from $E^{\prime}$ is denoted by $\sigma\left(E, E^{\prime}\right)\left(\beta\left(E, E^{\prime}\right)\right)$. Theorem 9 in this setting takes the following form.

Theorem 20. Suppose $\lambda$ is either $c_{0}$-factorable and monotone or has $\infty-$ GHP. Let $\sum_{j} x_{i j}$ be $\lambda$ multiplier convergent with respect to $\sigma\left(E, E^{\prime}\right)$ for each $i \in \mathbf{N}$. If

$$
\text { (\#) for each } t \in \lambda\left\{\sum_{j=1}^{\infty} t_{j} x_{i j}: i \in \mathbf{N}\right\} \text { is } \beta\left(E, E^{\prime}\right) \text { bounded, }
$$

then

(\#\#) for each $t \in \lambda$ the series $\sum_{j=1}^{\infty} t_{j} x_{i j}$ converge uniformly in $\beta\left(E, E^{\prime}\right)$ for $i \in \mathbf{N}$. 
A similar result is given in Theorem 2.32 of [Sw3].

We consider a form of the Hahn-Schur Theorem in the duality setting. For condition (\#) above if $\sigma\left(E, E^{\prime}\right)-\lim _{i} \sum_{j=1}^{\infty} t_{j} x_{i j}$ exists, then $\left\{\sum_{j=1}^{\infty} t_{j} x_{i j}: i \in \mathbf{N}\right\}$ is $\sigma\left(E, E^{\prime}\right)$ bounded and is $\beta\left(E, E^{\prime}\right)$ bounded if $E, E^{\prime}$ is a Banach-Mackey pair ([Wi] 10.4.3) so (\#) will be satisfied. Let $\mathcal{A}$ be a family of $\sigma\left(E^{\prime}, E\right)$ bounded subsets of $E^{\prime}$ which contains the finite sets and whose union is all of $E^{\prime}$ and let $\tau_{\mathcal{A}}$ be the polar topology of uniform convergence on the members of $\mathcal{A}$ ([K1] 21.7, [Sw1] Chapter 17, [Wi] 8.5). Then Theorem 14 will yield the following Hahn-Schur Theorem in this setting.

Theorem 21. Suppose $\lambda$ is either $c_{0}$-factorable and monotone or has $\infty-$ $G H P$ and that $E, E^{\prime}$ is a Banach-Mackey pair. Let $\sum_{j} x_{i j}$ be $\lambda$ multiplier convergent with respect to $\sigma\left(E, E^{\prime}\right)$ for each $i \in \mathbf{N}$. If for each $t \in \lambda$, $\sigma\left(E, E^{\prime}\right)-\lim _{i} \sum_{j=1}^{\infty} t_{j} x_{i j}$ exists and

$$
\text { (\&) for each } j \in \mathbf{N}, \tau_{\mathcal{A}}-\lim _{i} x_{i j}=x_{j} \text { exists, }
$$

then $\sum_{j} x_{j}$ is $\beta\left(E, E^{\prime}\right) \lambda$ multiplier convergent and $\tau_{\mathcal{A}}-\lim _{i} \sum_{j=1}^{\infty} t_{j} x_{i j}=$ $\sum_{j=1}^{\infty} t_{j} x_{j}$ for $t \in \lambda$.

We give an example showing the importance of condition $(\&)$ when $\tau_{\mathcal{A}}$ is the strong topology $\beta\left(E, E^{\prime}\right)$ and that the condition cannot be weakened to weak convergence. Of course, the condition is necessary for the conclusion in Theorem 21 to hold.

Example 22. Consider the dual pair $c_{0}, l^{1}$ and $\lambda=l^{1}$. Let $x_{i j}=e^{i} / 2^{j}$. The sequence $\left\{e^{i} / 2^{j}\right\}_{i}$ is weakly convergent to 0 but is not strongly $\left(=\|\cdot\|_{\infty}\right)$ convergent. The series $\sum_{j} x_{i j}$ is $l^{1}$ multiplier convergent with respect to $\|\cdot\|_{\infty}$ and for $t \in l^{1}, \sigma\left(c_{0}, l^{1}\right)-\lim _{i} \sum_{j=1}^{\infty} t_{j} x_{i j}=0$ but $\left\{\left\|\sum_{j=1}^{\infty} t_{j} x_{i j}\right\|_{\infty}\right\}$ doesn't converge to 0 if , for example, $t=\left\{1 / 2^{j}\right\}$.

Next, we give an example showing the importance of the gliding hump assumptions on the multiplier space $\lambda$ in Theorem 9 .

Example 23. Consider the dual pair $l^{\infty}, l^{1}$ and $\lambda=l^{\infty}$. Let

$$
\left\{x_{i j}\right\}_{j}=\left\{e^{1}, e^{2}, \ldots, e^{i}, 0,0, \ldots\right\} .
$$

The sequences $\left\{x_{i j}\right\}_{i}$ are eventually constant and therefore , $\beta\left(l^{\infty}, l^{1}\right)=$ $\|\cdot\|_{\infty}$ convergent. For each $t \in \lambda=l^{\infty},\left\{\sum_{j=1}^{\infty} t_{j} x_{i j}: i\right\}=\left\{\sum_{j=1}^{i} t_{j} e^{j}: i\right\}$ is 
$\|\cdot\|_{\infty}$ bounded. However, the series $\sum_{j=1}^{\infty} t_{j} x_{i j}=\sum_{j=1}^{i} t_{j} e^{j}$ do not converge uniformly with respect to $\|\cdot\|_{\infty}$ if $t$ is the constant sequence $\{1\}$. Note that the multiplier space $l^{\infty}$ has the strong gliding hump property but does not satisfy the assumptions on the mutiplier space in Theorem 9.

We next give an application to a space of continuous functions. Let $S$ be a compact Hausdorff space and $X$ a normed space. Let $C_{X}(S)$ be the space of continuous functions $f: S \rightarrow X$ and assume $C_{X}(S)$ has the sup-norm, $\|f\|=\max \{\|f(s)\|: s \in S\}$. Define $b: C_{X}(S) \times S \rightarrow X$ by $b(f, s)=f(s)$; the topology $w\left(C_{X}(S), S\right)$ is the topology of pointwise convergence on $S$ and condition $\left(\gamma^{\prime}\right)$ is satisfied. Thomas has established an Orlicz-Pettis Theorem for subseries convergence with respect to the topology of pointwise convergence and the sup-norm topology ([Th]); a multiplier convergent version is given in [Sw3]4.68. We use Theorem 14 to establish a Hahn-Schur Theorem in this setting. In this setting the set $S$ is pointwise bounded and Theorems 9 and 14 yield the following results.

Theorem 24. Suppose $\lambda$ is either $c_{0}$-factorable and monotone or has $\infty-$ $G H P$ and $\sum_{j} f_{i j}$ is $\lambda$ multiplier convergent with respect to $w\left(C_{X}(S), S\right)$ for $i \in \mathbf{N}$. If

$$
\text { (\#) for every } t \in \lambda\left\{\sum_{j=1}^{\infty} t_{j} f_{i j}: i \in \mathbf{N}\right\} \text { is }\|\cdot\|_{\infty} \text { bounded, }
$$

then

$$
\begin{gathered}
(\# \#) \text { for every } t \in \lambda \text { theseries } \sum_{j=1}^{\infty} t_{j} f_{i j} \text { converge } \\
\text { uniformly with respect to }\|\cdot\|_{\infty} \text { for } i \in \mathbf{N} \text {. }
\end{gathered}
$$

Theorem 25. (Hahn-Schur) Suppose $\lambda$ is either $c_{0}$-factorable and monotone or has $\infty-G H P, \sum_{j} f_{i j}$ is $\lambda$ multiplier convergent with respect to $w\left(C_{X}(S), S\right)$ for $i \in \mathbf{N}$ and for every $t \in \lambda, s \in S, \lim _{i} \sum_{j=1}^{\infty} t_{j} f_{i j}(s)$ exists. If (\#) holds and for every $j$ there exists $f_{j} \in C_{X}(S)$ such that $\lim _{i}\left\|f_{i j}-f_{j}\right\|_{\infty}=0$, then $\sum_{j} f_{j}$ is $\lambda$ multiplier convergent with respect to $w\left(C_{X}(S), S\right)$ and

$$
\lim _{i}\left\|\sum_{j=1}^{\infty} t_{j} f_{i j}-\sum_{j=1}^{\infty} t_{j} f_{j}\right\|_{\infty}=0 .
$$


The last result has the form of a Hahn-Schur Theorem in the sense that a weakly convergent series converges in a much stronger topology.

Finally, we give an application to spaces with a Schauder basis. Stiles established a remarkable Orlicz-Pettis Theorem for such spaces which was the first Orlicz-Pettis Theorem for non-locally convex spaces ([St]). Let $E$ be a topological vector space with a Schauder basis $\left\{b_{j}\right\}$ and coordinate functionals $\left\{f_{j}\right\}$ (i.e., each $x \in E$ has a unique expansion $x=\sum_{j=1}^{\infty} u_{j} b_{j}$ and $f_{j}: E \rightarrow \mathbf{R}$ is defined by $\left.f_{j}(x)=u_{j}\right)$. Let $P_{k}: E \rightarrow E$ be the projection defined by $P_{k}(x)=\sum_{j=1}^{k} f_{j}(x) b_{j}$ so $\lim _{k} P_{k}(x)=x$ with convergence in $E$. Define $b: E \times\left\{P_{k}\right\} \rightarrow E$ by $b\left(x, P_{k}\right)=P_{k} x$. If $F=\operatorname{span}\left\{f_{j}: j \in \mathbf{N}\right\}$, then $E, F$ form a dual pair and the weak topology $\sigma(E, F)$ is equal to $w\left(E,\left\{P_{k}\right\}\right)$; $\sigma(E, F)$ is the weak topology for Orlicz-Pettis Theorems considered by Stiles for subseries convergent series and was considered later for multiplier convergent series in [Sw3], 9.10. Since $\left\{P_{k}\right\}$ is pointwise bounded, Theorems 9 and 14 yield the following results in this setting.

Theorem 26. Suppose $\lambda$ is either $c_{0}$-factorable and monotone or has $\infty-$ $G H P$ and $\sum_{j} x_{i j}$ is $\lambda$ multiplier convergent with respect to $\sigma(E, F)$ for every $i \in \mathbf{N}$. If

$$
\text { (\#) for every } t \in \lambda\left\{\sum_{j=1}^{\infty} t_{j} P_{k} x_{i j}: i, k \in \mathbf{N}\right\} \text { is bounded, }
$$

then

$$
\begin{aligned}
& \text { (\#\#) for every } t \in \lambda \text { the series } \sum_{j=1}^{\infty} t_{j} x_{i j} \text { converge uniformly in } E \text { for } \\
& \qquad i \in \mathbf{N} .
\end{aligned}
$$

Proof : By Theorem 9 the series $\sum_{j=1}^{\infty} t_{j} P_{k} x_{i j}$ converge uniformly in $E$ for $i, k \in \mathbf{N}$. Let $U$ be a closed neighborhood of 0 in $E$. There exists $N$ such that $n \geq N$ implies $\sum_{j=n}^{\infty} t_{j} P_{k} x_{i j}=P_{k} \sum_{j=n}^{\infty} t_{j} x_{i j} \in U$ for $i, k \in \mathbf{N}$. Letting $k \rightarrow \infty$ gives $\sum_{j=n}^{\infty} t_{j} x_{i j} \in U$ for $n \geq N, i \in \mathbf{N}$ and, hence, the conclusion.

Similarly, Theorem 14 yields a Hahn-Schur Theorem.

Theorem 27. Suppose $\lambda$ is either $c_{0}$-factorable and monotone or has $\infty-$ $G H P, \sum_{j} x_{i j}$ is $\lambda$ multiplier convergent with respect to $\sigma(E, F)$ for every $i \in \mathbf{N}$, (\#) and for every $j$ there exists $x_{j} \in E$ such that 
(*) $\lim _{i} P_{k} x_{i j}=P_{k} x_{j}$ uniformly fork $\in \mathbf{N}$.

Then $\sum_{j} x_{j}$ is $\lambda$ multiplier convergent with respect to $\sigma(E, F)$ and for every $t \in \lambda \lim _{i} \sum_{j=1}^{\infty} t_{j} x_{i j}=\sum_{j=1}^{\infty} t_{j} x_{j}$ in $E$.

If $E$ is a complete quasi-normed space, then the $\left\{P_{k}\right\}$ are equicontinuous ([Sw1]10.1.14]) and condition (\#) of Theorem 26 can be replaced by $"\left\{\sum_{j=1}^{\infty} t_{j} x_{i j}: i \in b f N\right\}$ is bounded in $E "$ and condition $(*)$ in Theorem 27 can be replaced by the simpler condition " $\lim _{i} x_{i j}=x_{j}$ in $E "$.

\section{References}

[BCS] O. Blasco, J. M. Calabuig, T. Signes, A bilinear version of OrliczPettis theorem, J. Math. Anal. Appl., 348, pp. 150-164, (2008).

[CL] A. Chen, R. Li, A version of Orlicz-Pettis Theorem for quasihomogeneous operator space, J. Math. Anal. Appl., 373, pp. 127-133, (2011).

[Ga] D. J. H. Garling, The $\beta$ - and $\gamma$-duality of sequence spaces, Proc. Camb. Phil. Soc., 63, pp. 963-981, (1967).

[Ha] H. Hahn, Uber Folgen linearen Operationen, Monatsch. fur Math. und Phys., 32, pp. 1-88, (1922).

[K1] G. Kothe, Topological Vector Spaces I, Springer, Berlin, (1983).

[K2] G. Kothe, Topological Vector Spaces II, Springer, Berlin, (1979).

[LW] R. Li, J. Wang, Invariants in Abstract Mapping Pairs, J. Aust. Math. Soc., 76, pp. 369-381, (2004).

[Sc] J. Schur, Uber lineare Transformation in der Theorie die unendlichen Reihen, J. Reine Agnew. Math., 151, pp. 79-111, (1920).

[St] W. J. Stiles, On Subseries Convergence in F-spaces, Israel J. Math., 8, pp. 53-56, (1970).

[Sw1] C. Swartz, An Introduction to Functional Analysis, Marcel Dekker, N. Y., (1992). 
[Sw2] C. Swartz, Infinite Matrices and the Gliding Hump, World Sci. Publ., Singapore, (1996).

[Sw3] C. Swartz, Multiplier Convergent Series, World Sci. Publ., Singapore, (2009).

[Sw4] C. Swartz, A Bilinear Orlicz-Pettis Theorem, J. Math. Anal. Appl., 365, pp. 332-337, (2010).

[Th] G. E. F. Thomas, L'integration par rapport a une mesure de Radon vectorielle, Ann. Inst. Fourier, 20(, pp. 55-191, (1970).

[Wi] A. Wilansky, Modern Methods in Topological Vector Spaces, McGraw-Hill, N. Y., (1978).

Charles Swartz

Department of Mathematical Sciences

New Mexico State University

Las Cruces, NM 88003,

U. S. A.

e-mail : cswartz@nmsu.edu 